\title{
Buruli ulcer: reductive evolution enhances pathogenicity of Mycobacterium ulcerans
}

\section{Caroline Demangel*\|, Timothy P. Stinear ${ }^{\ddagger \|}$ and Stewart T. Cole ${ }^{\S}$}

Abstract | Buruli ulcer is an emerging human disease caused by infection with a slow-growing pathogen, Mycobacterium ulcerans, that produces mycolactone, a cytotoxin with immunomodulatory properties. The disease is associated with wetlands in certain tropical countries, and evidence for a role of insects in transmission of this pathogen is growing. Comparative genomic analysis has revealed that $M$. ulcerans arose from Mycobacterium marinum, a ubiquitous fast-growing aquatic species, by horizontal transfer of a virulence plasmid that carries a cluster of genes for mycolactone production, followed by reductive evolution. Here, the ecology, microbiology, evolutionary genomics and immunopathology of Buruli ulcer are reviewed.

\section{Virulence plasmid An autonomously replicating DNA element that is found in some pathogenic \\ microorganisms and harbours genes that are essential for that microorganism to cause disease.}

The human skin disease that results from infection with Mycobacterium ulcerans is commonly known as Buruli ulcer (BU), but would have been called Bairnsdale ulcer if microbiological history had been strictly respected. In 1935, a series of unusual, painless ulcers in patients from a remote farming community in the Bairnsdale district of south-east Australia was reported ${ }^{1}$. Some 13 years later, Australian researchers discovered the aetiological agent of Bairnsdale ulcer, a previously unknown mycobacterium that they named M. ulcerans ${ }^{2}$. During the 1960s, many cases of infection with $M$. ulcerans were reported in Uganda, particularly in Buruli County (now known as the Nakasongola district), and thus the disease became more generally known as BU. Today, the disease is far more widespread in West and Central Africa, especially among impoverished rural communities, although other parts of the world are also affected. Thirty countries, mainly in the tropics, have reported cases of BU and in some settings, such as Ghana or Benin, $\mathrm{BU}$ is now more prevalent than leprosy ${ }^{3}$.

During the past 10 years, there has been considerable progress in our understanding of the ecology, aetiology and microbiology of $\mathrm{BU}$, which has led to better definition of risk factors and awareness of the potential role of insects in transmission of the disease. Comparative mycobacterial genomics has underpinned these advances and provided compelling evidence for the emergence of M. ulcerans as a pathogen through horizontal gene transfer of a virulence plasmid. Here, we review the current state of our knowledge and comment on prospects for disease control.

\section{Physiopathology and medical interventions}

M. ulcerans produces mycolactone, a macrolide cytotoxin with immunosuppressive properties that is present in the extracellular matrix surrounding large clusters of M. ulcerans cells organized as a biofilm ${ }^{4}$. BU begins as a small, painless, raised skin papule, nodule, plaque or oedema. Osteomyelitis may occur in bone adjacent to a cutaneous lesion. Later, destruction of the subcutaneous adipose tissue leads to collapse of the epidermis and formation of a characteristic ulcer with undermined edges $^{5-7}$ (FIG. 1). Advanced lesions display massive tissue destruction and minimal inflammation, with extracellular microcolonies of M. ulcerans in the superficial necrotic areas ${ }^{8}$. Despite some anti-phagocytic activity of mycolactone, phagocytes can internalize M. ulcerans in vitro, although this culminates in host cell death ${ }^{9,10}$. In contrast to wild-type strains, mycolactone-deficient mutants generate inflammation in the guinea pig model ${ }^{11}$, suggesting that production of mycolactone by intracellular bacteria can suppress innate inflammatory responses to infection in vivo.

The physiopathology of BU is closely associated with diffusion of the toxin from bacterial foci, as first suspected from histopathological studies of infected tissues $^{12}$. Injection of mycolactone is sufficient to induce 


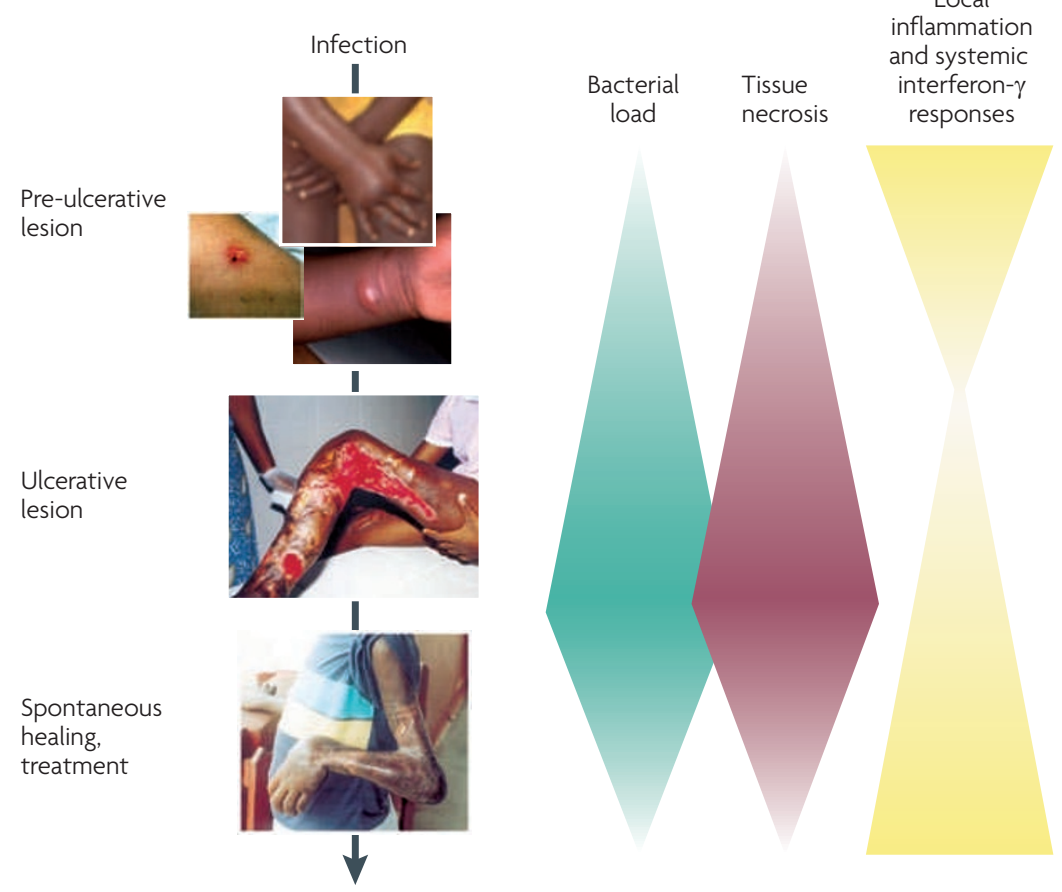

Figure 1 | Disease progression. The progression from the pre-ulcerative to the ulcerative and healing stages of Buruli ulcer disease is shown. The width of the green, red and yellow shapes denotes the extent of the progression for bacterial load, necrosis and inflammatory responses, respectively.

Schwann cell

A cell that surrounds neurons, produces myelin in nerves in the peripheral nervous system and is the preferred niche for the leprosy bacillus, a relative of Mycobacterium ulcerans. Schwann cells were named after their discoverer, Theodor Schwann

Secondary infection An infection that occurs as a consequence of a weakened or perturbed immune response following a primary infection by a different pathogen.

Insertion sequence A mobile DNA element that encodes at least one transposase which is flanked by short inverted repeats that permit the element to copy itself within or between chromosomes. necrotic lesions in the skin of guinea pigs ${ }^{13}$, by an apoptosis-dependent mechanism ${ }^{14}$. Consistent with the extensive tissue damage observed, mycolactone kills a range of mammalian cells ${ }^{15}$, but the molecular basis of mycolactone-induced apoptosis nevertheless remains unclear. Indeed, studies that used mouse fibroblasts in vitro indicate that apoptosis follows cytopathic effects, including cytoskeletal rearrangements and cell-cycle arrest $^{13,14}$.

Although M. ulcerans displays no tropism for Schwann cells, nerve invasion occurs at the perineural and endoneural levels, causing degeneration ${ }^{16}$. Similar alterations are induced by injecting purified toxin into mice ${ }^{17}$, suggesting that mycolactone diffuses into Schwann cells in vivo to cause the painlessness that characterizes the disease. A surprising feature of $\mathrm{BU}$ is the scarcity of secondary infections of these immunosuppressed open wounds, which raises the possibility that $M$. ulcerans produces secondary metabolites with anti-microbial activity (WHO provides a method for diagnosing secondary bacterial infection in M. ulcerans disease; see Further information).

$\mathrm{BU}$ is usually diagnosed on the basis of clinical findings, occasionally confirmed by microscopy, but to treat disease more effectively it is essential to develop novel, field-friendly diagnostic approaches to allow earlier identification of patients. The extensive cross-reactivity among mycobacterial antigens complicates the use of specific serological assays ${ }^{18}$, although the unique ability of $M$. ulcerans to produce mycolactone makes this toxin an attractive candidate for the development of diagnostic tests. However, this hydrophobic molecule is not immunogenic, and attempts to raise anti-mycolactone antibodies have been unsuccessful.

Treatment of BU remains complicated and generally requires both surgery, sometimes accompanied by skin grafts, and prolonged courses of antibiotics. WHO recommends that a combination of rifampicin (oral) and streptomycin (intramuscular) be administered daily for 8 weeks ${ }^{19}$. However, although results from this treatment are encouraging ( 4 weeks of treatment leads to culture negativity), most patients need to be hospitalized owing to the frequency, duration and route of antibiotic administration. New drugs are clearly needed to treat this disease and better biomarkers are required to monitor the therapeutic response of patients.

There is no vaccine against $M$. ulcerans, although significant, but short-lasting, protection against BU has been reported following immunization with the Mycobacterium bovis bacille Calmette-Guérin (BCG) vaccine $^{20}$. Prospects for vaccine development include modified BCGs and subunit-based vaccines ${ }^{21}$. DNA vaccines that encode antigen $85 \mathrm{~A}$ and the heat-shock protein Hsp65 have been shown to protect mice against $M$. ulcerans infection ${ }^{22-25}$. However, these vaccines remain less protective than BCG in mice, even when administered in DNA-prime-boost protocols. Comparative genomics could open new avenues for vaccine research and improved diagnostics.

\section{Epidemiology, risk factors and insect vectors}

The epidemiology of $\mathrm{BU}$ is poorly understood and outbreaks are sporadic and unpredictable; however, proximity to, or contact with, slowly flowing or stagnant watercourses is a recognized risk factor ${ }^{26-28}$. Furthermore, disease is often confined to specific areas and has sometimes appeared following major environmental changes, such as deforestation, flooding, or the introduction of dams and irrigation systems ${ }^{29}$. In Benin, for example, the incidence of $\mathrm{BU}$ is tenfold greater in areas that have undergone environmental perturbation compared with control areas ${ }^{29}$.

In these settings, M. ulcerans is associated with aquatic vegetation, such as plants and algae ${ }^{30}$, whereas snails and other organisms that feed on this vegetation can serve as passive hosts ${ }^{31}$. Portaels and colleagues were the first to use PCR-based detection methods to investigate a role for insect vectors in transmission ${ }^{32}$. Other groups have performed similar environmental surveys to uncover likely niches of $M$. ulcerans. M. ulcerans from environmental specimens is notoriously difficult and unreliable to culture owing to the long generation time of the pathogen ( $>48$ hours) and overgrowth by contaminants, including other mycobacteria. PCR is more reliable but is not foolproof, as one of the diagnostic PCR targets, insertion sequence (IS) 2404, is not restricted to M. ulcerans. Other targets also reside on the virulence plasmid, and the plasmid can be transferred to other species ${ }^{33}$. Such PCR methods have been used to detect $M$. ulcerans in aquatic samples, including water, mud, plants, insects, molluscs and fish, from endemic areas. 


\section{Raptorial legs}

The two powerful front legs of some insect species that have evolved to catch and hold prey.

Photochromogenic pigment A distinctive, bright yellow carotenoid pigment that is produced owing to exposure to light.

Dichotomous lifestyle The ability to live in two different environments; for example, the ability to inhabit diverse aquatic ecosystems as an extracellular bacterium as well as different intracellular niches, such as within amoebae or host macrophages.

Granulomatous disease The pathology associated with the distinctive, organized cellular immune response following mycobacterial infection.

Multilocus sequence analysis

A system for studying the population structure of bacterial populations by comparing the DNA sequences from a set of conserved genes among different strains.
An important series of experiments performed by Marsollier and colleagues ${ }^{34}$ showed that M. ulcerans is carried and multiplies in the water bug, Naucoris cimicoides. Using two different methods of infection, either feeding $N$. cimicoides with larvae containing M. ulcerans or directly injecting the bacteria, these investigators showed not only that the bacterium became established in $N$. cimicoides but also that the transmission of M. ulcerans to laboratory mice occurred through biting, leading to the appearance of necrotic lesions that were reminiscent of $\mathrm{BU}$ in humans ${ }^{34}$.

Details are available of the trafficking of $M$. ulcerans through $N$. cimicoides in captivity ${ }^{35,36}$. The plasmatocytes in the coelomic cavity of the water bug phagocytose M. ulcerans and, as part of their circulatory process, transport the bacteria to the salivary glands where large-scale multiplication occurs ${ }^{35}$. Only toxin-producing $M$. ulcerans isolates can invade the salivary glands, and mycolactone is therefore key to both the early and long-term establishment of $M$. ulcerans in members of the Naucoridae family. Later, the raptorial legs of the insect are covered by biofilms that contain $M$. ulcerans, which could be important for transmission of the disease without biting ${ }^{35,36}$.

$N$. cimicoides is a carnivorous organism that preys on other insects, snails and small fish, but it does not normally bite humans, which weakens the possibility that biting is the main route of infection of humans. However, in a recent serological survey of healthy individuals in a BU-endemic region, high titres of antibodies were found to $N$. cimicoides salivary proteins ${ }^{37}$, indicating that humans can indeed be bitten. Importantly, immunization of mice with saliva from uninfected $N$. cimicoides conferred protection against subsequent infection with M. ulcerans from contaminated water bugs.

In a landmark study from Benin, M. ulcerans was isolated from the water strider (Gerris sp. from the aquatic order Hemiptera) in pure culture. Following isolation of the pathogen and infection of mice, the M. ulcerans strain was shown to be genetically and phenotypically identical to that isolated from patients living in the same region $^{38}$. However, the water strider, like the Naucoridae, generally avoids humans.

During the past decade, there have been several outbreaks of BU disease, both in residents and visitors, in parts of south-east Australia, and this provided an ideal setting to study disease transmission. Using PCR, a strong association was found between M. ulcerans and mosquitoes, predominantly Aedes camptorhynchus ${ }^{39}$. The incidence of BU rose in the spring and summer, and was followed by a cluster of human cases in the autumn and winter months. From this survey, it was estimated that the incubation period for BU was 3-4 months ${ }^{39}$. Furthermore, a bimodal pattern was observed, in which peaks represented young children and the elderly; this was similar to the pattern observed in West Africa, with the exception that in south-east Australia the disease was less common in children ${ }^{40,41}$. One possible explanation for this difference lies in the more pronounced exposure to possible insect vectors, and the accompanying immunity to M. ulcerans, among the adult African population $^{37}$.
A parallel case-control study revealed that the risk of BU was halved in the group that took precautions against insect bites by applying insect repellent, wearing protective clothing or washing minor skin wounds $\mathrm{s}^{42}$. This strongly suggests that $M$. ulcerans is transmitted to humans by mosquito bites but, in turn, raises questions as to how the mosquitoes themselves become infected. Such contamination could occur at the larval stage from the water where the mosquitoes breed or through blood meals from other infected hosts. The conclusions of an extensive case-control study in a BU-endemic region of Cameroon are also consistent with transmission through insect bites, as protection was observed if bed nets were used in the home ${ }^{43}$. However, a large temporal and spatial survey conducted across Ghana of 15 BU-endemic and 12 BU-non-endemic areas revealed no association between biting hemipterans and M. ulcerans ${ }^{44}$. From these and other epidemiological studies, there is little evidence for person-to-person transmission. In summary, although there is now a substantial body of evidence to indicate an association between $M$. ulcerans and different insects that share the same aquatic ecosystem, the actual means of transmission to humans remains elusive. Here, the availability of new molecular and immunological tools should help.

\section{Genetically similar, phenotypically distinct}

Despite their contrasting phenotypes, M. ulcerans and $M$. marinum have almost identical genome sequences (FIG. 2a). M. ulcerans replicates slowly and produces no photochromogenic pigments. By contrast, $M$. marinum doubles every 6-11 hours and produces no mycolactone, but is photochromogenic and has a dichotomous lifestyle: M. marinum lives in diverse aquatic niches as well as a range of different intracellular environments, from freeliving aquatic amoebae and fish to macrophages from both frogs and humans ${ }^{45}$. In aquatic species, M. marinum causes a disseminated granulomatous disease that resembles dermal infection with Mycobacterium tuberculosis ${ }^{46}$. In humans, however, it provokes relatively minor granulomatous skin lesions, usually on the cooler extremities of the body.

Early phylogenetic work that applied multilocus sequence analysis (MLSA) revealed a clear delineation between strains of $M$. ulcerans and M. marinum, and indicated that all $M$. ulcerans strains have evolved from a common M. marinum progenitor ${ }^{47-50}$. MLSA also highlighted phylogeographical clonality among $M$. ulcerans strains (FIG. 2b), in which isolates from several African countries, South East Asia and both northern and south-eastern Australia belonged to three distinct genotypes, whereas strains from Japan and China were represented by two closely related genotypes and strains from Mexico and Suriname were represented by distinct genotypes ${ }^{50,51}$. These data have been corroborated and refined by microarray analyses ${ }^{52,53}$. Structural differences in mycolactone are also observed in strains of different genotype. Interestingly, the M. ulcerans isolates from South East Asia and Australia are more closely related to the African genotype than to strains from elsewhere. The lack of sequence diversity among 


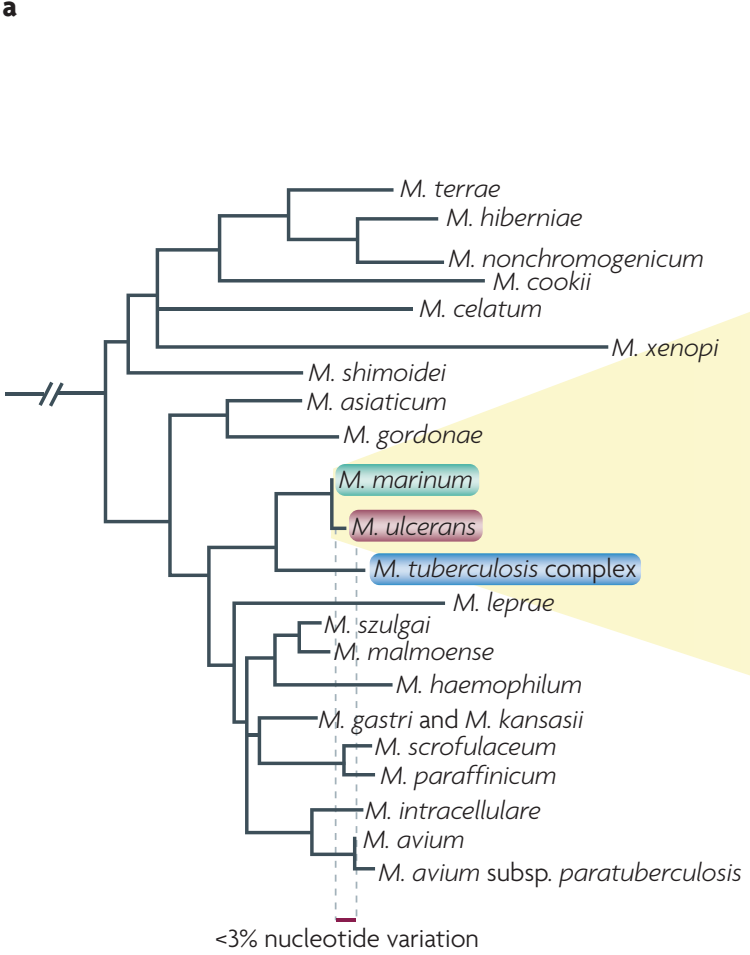

16S rRNA gene b

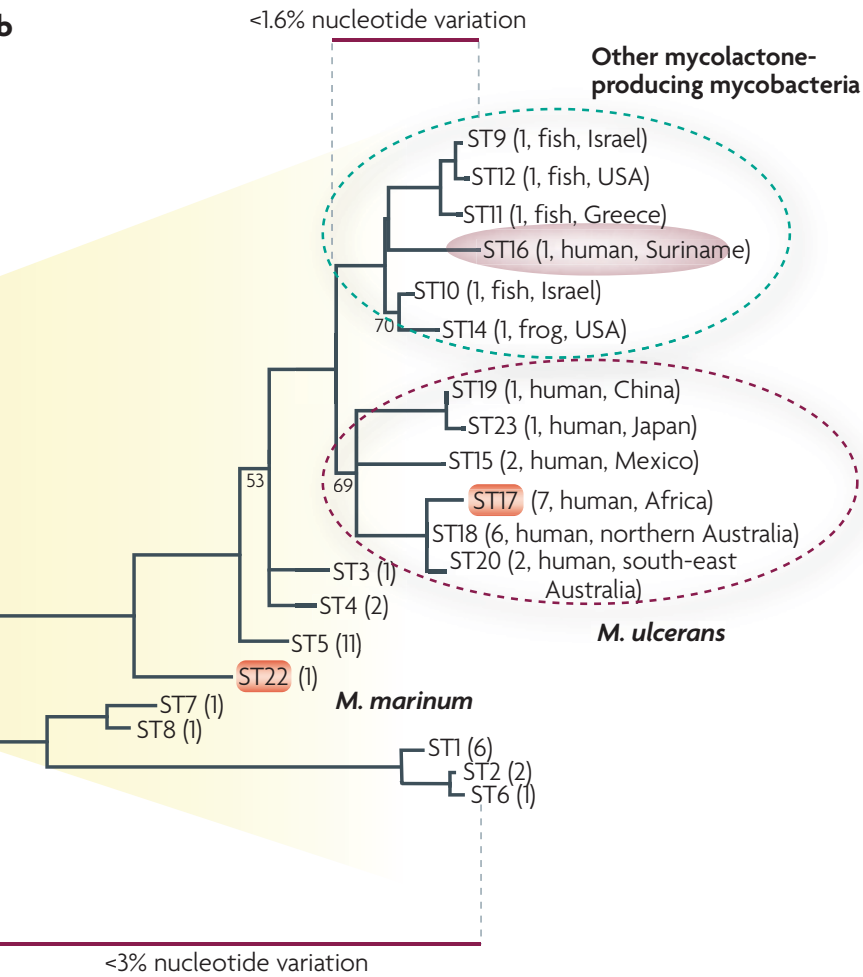

Multilocus sequence analysis

(3,210 bp crtB, adk, fbpA, aroE, groE, groL, ppk, sod and glcB)

c

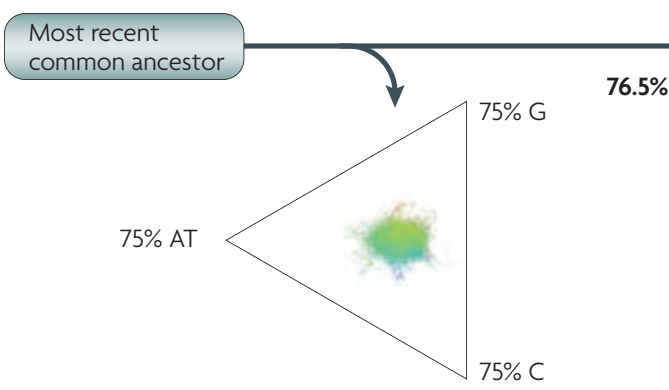

M. smegmatis

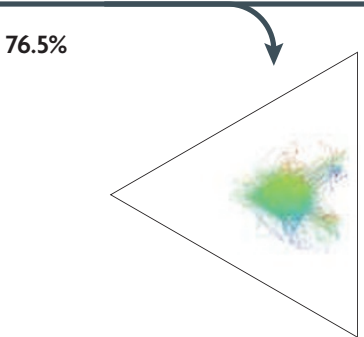

M. tuberculosis

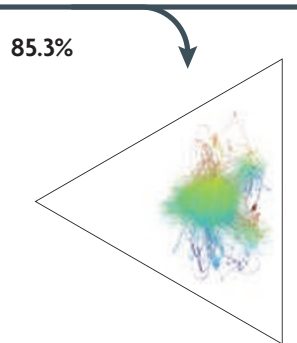

M. marinum

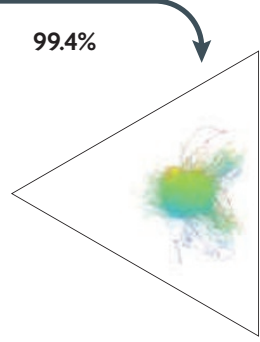

M. ulcerans

Figure 2 | Phylogenetic analysis and evolutionary scenario for Mycobacterium ulcerans, Mycobacterium marinum and Mycobacterium tuberculosis inferred from genomics. a | 16S rRNA phylogeny of the slow-growing mycobacteria, showing the relationship of M. ulcerans (highlighted in red) and M. marinum (highlighted in green) to M. tuberculosis (highlighted in blue) and other mycobacterial pathogens ${ }^{101}$. The purple line indicates defined clusters, such as M. marinum, M. ulcerans and the mycolactone-producing mycobacteria. $\mathbf{b} \mid$ The evolutionary history of M. marinum, $M$. ulcerans (indicated by a dashed red oval) and other mycolactone-producing mycobacteria (indicated by a dashed green oval) was inferred using the neighbour-joining method ${ }^{102}$ from multilocus sequence data ${ }^{33}$. The bootstrap consensus tree was inferred from 1,000 replicates and was assumed to represent the evolutionary history of the taxa analysed ${ }^{103}$. Branches that correspond to partitions reproduced in less than $50 \%$ of the replicates were collapsed. The tree is drawn to scale, and branch lengths represent the evolutionary distances used to infer the phylogenetic tree. The first, second and third codon positions were included, and a total of 3,210 positions were included in the final data set. Phylogenetic analyses were conducted in MEGA4 (molecular evolutionary genetics analysis 4$)^{104}$. Complete genome sequences were available for strains of the sequence types (STs) highlighted in red; the number of genome sequences available and their phylogenetic and geographical origin are indicated in brackets. The strain isolated from humans is indicated in pink. The yellow triangle shows that part $\mathbf{b}$ is an expansion of a section of the tree shown in part $\mathbf{a}$. $\mathbf{c}$ / Whole-genome DNA-composition analyses of four mycobacterial genomes generated with gene spaghetti, a method for visualizing DNA base composition variation and the use of codons in a genome. The gradient of colours reflects the AT skew $(T-A) /(T+A)$, from $60 \% T$-rich (dark red) to $60 \%$ A-rich (dark blue). The florid gene-spaghetti patterns for M. ulcerans, M. marinum and M. tuberculosis reflect the important contribution of lateral gene transfer to the evolution of these species compared with M. smegmatis. The average percentage amino-acid identity between each species is also indicated (in bold) for a core set of 1,072 orthologous coding DNA sequences that were identified by whole genome comparisons between these species. 
Table 1 | Genomic comparisons of Mycobacterium marinum and Mycobacterium ulcerans

\begin{tabular}{|c|c|c|}
\hline & M. marinum $\mathbf{M}$ & M. ulcerans Agy99 \\
\hline $\begin{array}{l}\text { Genome size and } \\
\text { arrangement }\end{array}$ & $\begin{array}{l}\text { - Circular chromosome; } 6,637 \mathrm{~kb} \\
\text { - Circular mercury resistance plasmid } \\
\text { (pMM23; } 23 \mathrm{~kb} \text { ) }\end{array}$ & $\begin{array}{l}\text { - Circular chromosome; } 5,632 \mathrm{~kb} \\
\text { - Circular mycolactone-associated plasmid } \\
\text { (pMUM001; } 174 \mathrm{~kb} \text { ) }\end{array}$ \\
\hline Number of genes & 5,424 & 4,160 \\
\hline $\begin{array}{l}\text { Number of } \\
\text { pseudogenes }\end{array}$ & 65 & 771 \\
\hline $\begin{array}{l}\text { Number of insertion } \\
\text { sequences (ISs) }\end{array}$ & $\begin{array}{l}7 \text { ISs; Myma01 (7 copies), Myma02 (7 copies), } \\
\text { Myma03 ( } 4 \text { copies), Myma04 (5 copies) and } \\
\text { Myma05, Myma06 and Myma07 ( } 2 \text { copies of } \\
\text { each) }\end{array}$ & IS2404 (213 copies) and IS2606 (91 copies) \\
\hline $\begin{array}{l}\text { Number of PE and } \\
\text { PPE genes }\end{array}$ & 175 PE genes and 106 PPE genes & 69 PE genes and 46 PPE genes \\
\hline $\begin{array}{l}\text { Number of ESX } \\
\text { secretion systems }\end{array}$ & 5 & 3 \\
\hline $\begin{array}{l}\text { Number of ESX } \\
\text { effectors }\end{array}$ & 18 espA paralogues and 31 esx paralogues & 2 espA paralogues and 14 esx paralogues \\
\hline $\begin{array}{l}\text { Number of } \\
\text { phospholipase C } \\
\text { genes }\end{array}$ & $\begin{array}{l}\text { Seven phospholipase } C \text { paralogues (plcB, } \\
\text { plcB1, plcB2, plcB3, plcB4, plcB5 and plcB6) }\end{array}$ & $\begin{array}{l}\text { One gene that encodes phospholipase C } \\
\text { (plcB); others have become pseudogenized } \\
\text { or lost by DNA deletion }\end{array}$ \\
\hline $\begin{array}{l}\text { Number of } \\
\text { lipoproteins }\end{array}$ & 88 genes that encode lipoproteins & $\begin{array}{l}77 \text { genes that encode lipoproteins; lipY has } \\
\text { been lost by deletion }\end{array}$ \\
\hline Phenolic glycolipids & Mycoside M & $\begin{array}{l}\text { Phenolphthiodiolone lipid backbone cannot } \\
\text { be glycosylated as the glycosyl transferase } \\
\text { (MUL_1998) has been pseudogenized }\end{array}$ \\
\hline Mycolactone & Not produced & Mycolactone A and B \\
\hline
\end{tabular}

the African isolates suggests that this M. ulcerans clone spread recently throughout Africa. The availability of complete genome sequences from an African epidemic strain of M. ulcerans (strain Agy99, isolated from Ghana in 1999) and a strain of clinical M. marinum (strain M) confirmed this evolutionary scenario, highlighting how horizontal gene transfer and reductive evolution have remodelled an $M$. marinum progenitor into M. ulcerans (FIG. 2C).

In silico genomic comparisons of $M$. ulcerans with $M$. marinum confirmed the close genetic relationship between these species, as they shared more than 4,000 orthologous and syntenic protein-coding DNA sequences (CDSs) (TABLE 1) and had an average sequence identity of $98.3 \%$. This analysis also revealed that $M$. ulcerans had lost over 1.1 Mb of DNA owing to deletions (TABLE 1), whereas $168 \mathrm{~kb}$ had been acquired by M. marinum, mostly in the form of 10 prophages. Also evident were many chromosome rearrangements that were facilitated, at least in part, by the high number of IS2404 (213 copies) and IS2606 (91 copies), which disrupt $>110$ genes $^{54}$. All M. ulcerans strains tested have 11 chromosomal CDSs that seem to be specific to this bacterium and might, in conjunction with mycolactone, contribute to the pathology associated with BU. These genes, and their products, could also be used to develop new diagnostic tests ${ }^{54}$.

M. ulcerans has thus evolved through lateral gene transfer and reductive evolution, acquisition of the virulence plasmid pMUM001 (the role of which is discussed below), massive expansion of IS2404 and IS2606, extensive pseudogene formation, genome rearrangements and gene deletion. Like Yersinia pestis ${ }^{55}$ and Bordetella pertussis $^{56}, M$. ulcerans has all the characteristics of a bacterium that has recently passed through an evolutionary bottleneck and is adapting to a new niche environment. The challenge remains to find that niche, and in this respect, genomics may hold some clues.

\section{Loss or gain of virulence and immunogenicity?}

The trend that has emerged from reductive evolution in M. ulcerans is the loss of many virulence factors and immunogens compared with its $M$. marinum progenitor (TABLE 1) and the gain of an immunosuppressive cytotoxin (FIG. 3). Most noteworthy is the drastic reduction in the cell surface proteins PE and PPE from 281 in M. marinum to 115 in $M$. ulcerans, accompanied by depletion of the related ESX secretion systems and their effector proteins. $\mathrm{PE}$ and PPE proteins have characteristic amino-terminal domains and biased amino-acid content, as they are particularly rich in glycine and alanine. They are restricted to mycobacteria, where they are found in the cell envelope, but their precise function is unknown. The ESX loci, which encode type VII secretion systems ${ }^{57}$, are required to export members of the ESAT-6 (6 kDa early secretory antigenic target) protein family and specific effectors, such as EspA (ESX-1 secretion-associated protein $\mathrm{A})^{58}$. In at least one case, ESX-1, they are major contributors to mycobacterial virulence.

A few examples merit comment. In M. marinum, $\underline{L i p Y}$ is an immunodominant PPE protein with triacylglycerol hydrolase activity ${ }^{59}$, yet its gene has been deleted by $M$. ulcerans. In another example, the ESX secretome 


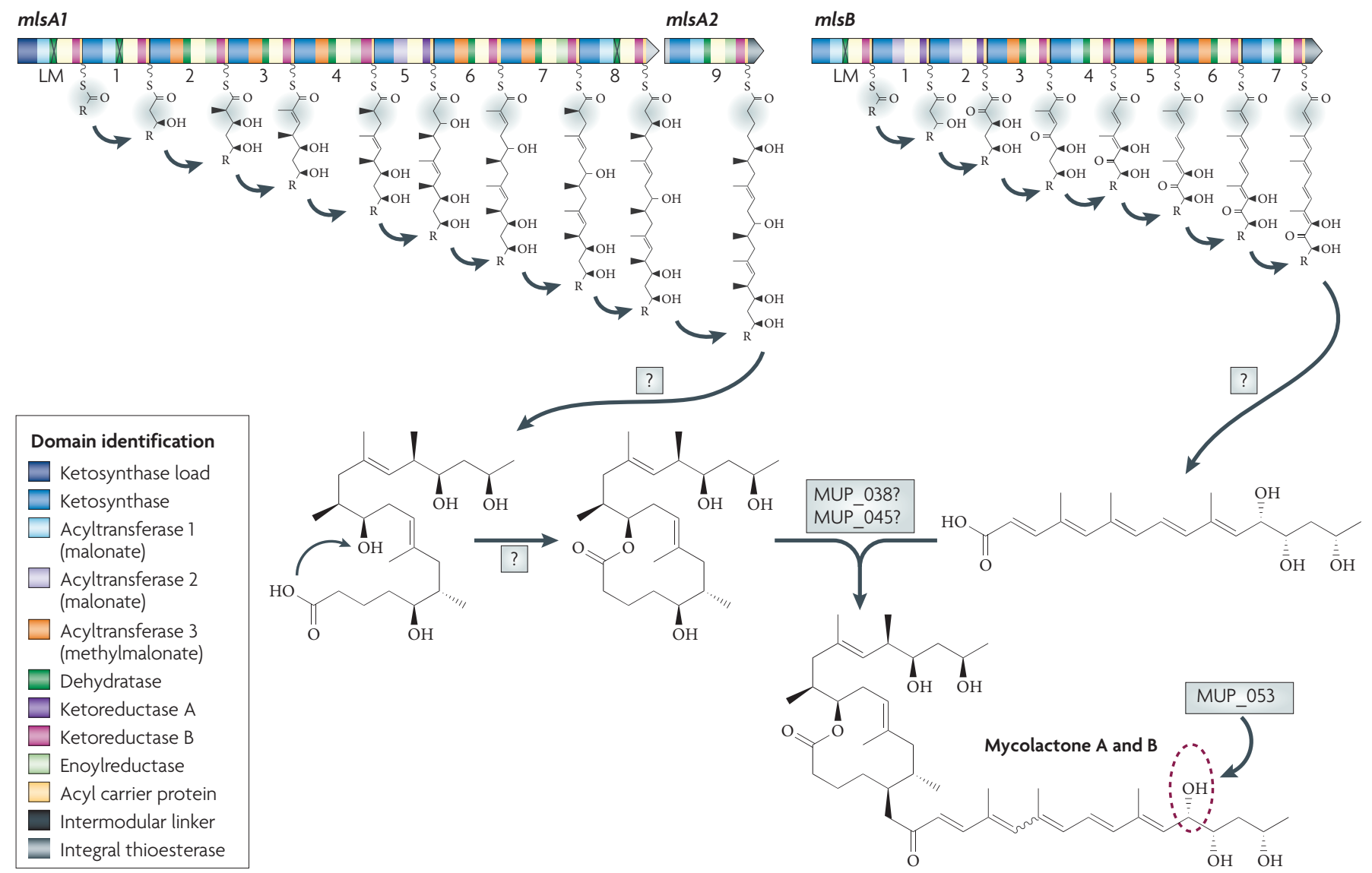

Figure 3 | Proposed pathway for the biosynthesis of mycolactone A and B. The mls cluster and accessory gene arrangement of pMUM001, as well as the domain and module organization of the mycolactone polyketide synthase (PKS) genes, are shown. The different domains of each of the three Mls proteins (MlsA1, MlsA2 and MlsB) are represented by the coloured blocks described in the key. Module arrangements are depicted below each protein. The module responsible for a particular chain extension and the modified substrate are shown tethered to the acyl carrier protein domain of each extension module. Key steps in the biosynthesis pathway are shown and the enzyme involved is indicated if known. A question mark indicates that the enzymes involved have not yet been identified or in the case of MUP_038 and MUP_045 have not been definitively proven. The dashed red circle indicates that the hydroxyl group is catalysed by MUP_053. LM, loading module.

was depleted from five systems to three ${ }^{54}$. Consequently, the protein substrates these systems export - including potent T-cell antigens, such as ESAT-6, which have important roles in granuloma formation and other aspects of pathogenesis ${ }^{58}$, and the EspA effector proteins ${ }^{60}$, which have been reduced from 18 paralogues in M. marinum to two in M. ulcerans - will be missing or less abundant. A recent study examined a wide range of $M$. ulcerans strains for disruption to the ESX loci, and found that although some strains exhibit the same pattern as M. ulcerans Agy99, others have acquired independent deletions or loss-of-function mutations in these regions. This suggests that loss of ESX loci provides a selective advantage for mycolactone producers ${ }^{61}$ or, alternatively, that gain of a powerful cytotoxin renders these virulence factors superfluous.

Phenolic glycolipids (PGLs) are abundant cell-wall components, antigens and major virulence factors for several mycobacterial pathogens and can modulate innate immunity ${ }^{62}$. In M. marinum, PGLs are synthesized by glycosylation of a highly apolar and abundant polyketide-derived methyl-branched lipid intermediate called phenolphthiodiolone. M. ulcerans also synthesizes prodigious quantities of phenolphthiodiolone but cannot make PGL, as the gene for the glycosyl transferase that adds the rhamnosyl moiety (locus tag MUL_1998) is inactive $\mathrm{e}^{54,63}$. These observations are summarized in TABLE 1.

Other gene losses from $M$. ulcerans give some clues as to its habitat. For example, phytoene dehydrogenase, encoded by $\operatorname{crtI}$, is an essential enzyme for the biosynthesis of light-inducible carotenoid pigments by M. marinum. These pigments protect the bacterium from damage following exposure to sunlight ${ }^{64}$. M. ulcerans has an identical pigment locus to $M$. marinum but its crtI gene has a premature stop codon, suggesting that its pigments are not required for survival, presumably because the bacterium is not exposed to sunlight. Another observation from the M. ulcerans genome is that the pathways for anaerobic respiration have been 
lost, indicating that the bacterium might occupy an aerobic or microaerophilic environment. Together, these data provide a profile of a bacterium that has adapted to a dark and aerobic environment where slow growth, decreased ESX-mediated virulence and production of mycolactone provide an advantage for survival. This profile also suggests that we may need to think more broadly and look more widely than aquatic invertebrates to find the true reservoir of this pathogen.

\section{Mycolactone production}

A major finding from the $M$. ulcerans genome project was the virulence plasmid pMUM001 (REFS 65,66), which carries a cluster of three large genes $(m l s A 1, m l s A 2$ and $\underline{m l s B}$, which are $51 \mathrm{~kb}, 7.6 \mathrm{~kb}$ and $43 \mathrm{~kb}$ in size, respectively) that encode type I polyketide synthases (PKSs) (FIG. 3). Like type I fatty acid synthases, type I PKSs contain the multiple enzymatic activities that are required for one round of chain extension and modification in a single polypeptide. Detailed analysis of their predicted module and domain structure strongly suggested that these PKSs produce mycolactone, and this was subsequently confirmed by transposon mutagenesis ${ }^{66}$.

Together, MlsA1 and MlsA2 constitute a loading module and nine extension modules that synthesize the macrolactone core and upper side chain, whereas MlsB, with its loading module and seven extension modules, produces the acyl side chain. The proposed biosynthesis pathway for mycolactone is shown in FIG. 3, and highlights the sequential incorporation and modification of either acetate or propionate subunits at each extension module. pMUM001 also harbours three additional CDSs that encode putative auxiliary enzymes for mycolactone synthesis (FIG. 3). Cyp140A7 is a cytochrome $\mathrm{P} 450$ hydroxylase that probably hydroxlates C-12' of the mycolactone side chain ${ }^{67-69}$. Experimental evidence is lacking for the remaining two enzymes, a type II thioesterase (locus tag MUP_038) and a FabHlike ketosynthase (locus tag MUP_045), but they may play parts in chain termination and transfer of the mycolactone acyl side chain to the core ${ }^{15}$ (FIG. 3).

There are only a few reports of biochemical studies of the mycolactone PKS. The ketoreductase domains have been expressed and their enzymatic function confirmed ${ }^{70}$. A similar study with the integral thioesterase domains of MlsA2 and MlsB ${ }^{71}$ (FIG. 3), which may release the mature polyketide chain, revealed little activity, suggesting that these domains may be inactive in the Mls complex $^{71}$. A proteomic investigation identified MlsA1, MlsB, Cyp140A7 and MUP_045 in association with both the cytoplasmic and membrane fraction, indicating that synthesis occurs close to the membrane, which could facilitate mycolactone export ${ }^{72}$ (FIG. 3).

Different strains of M. ulcerans, and close relatives from fish and frogs, produce at least five structurally distinct mycolactones, named mycolactone A/B, C, D, E and $\mathrm{F}^{73-77}$. M. ulcerans strains from Africa, Malaysia and Japan produce mycolactone A/B, Australian strains produce mycolactone $\mathrm{C}$, Chinese strains produce mycolactone $\mathrm{D}$ and Mycobacterium liflandii and Mycobacterium pseudoshottsii produce the unique mycolactones $\mathrm{E}$ and $\mathrm{F}$, respectively ${ }^{15}$. The mycolactone type produced by South American strains is unknown. Immunosuppressive and cytotoxic activity measurements revealed a convenient 'alphabetical' gradient in which mycolactone A/B is the most potent and mycolactone $\mathrm{F}$ is the least potent ${ }^{67}$. Interestingly, all mycolactones have a conserved core structure, and any variation occurs in the length, methyl branching, oxidation state and stereochemistry of the acyl side chain ${ }^{15}$.

\section{Mycolactone-producer evolution}

Mycolactone-producing mycobacteria have been recovered from fish and frogs around the world, but so far have not been associated with human disease. Based on minor phenotypic differences, they were given species names, such as $M$. pseudoshottsii, $M$. liflandii and M. marinum ${ }^{15,49,69,73,78}$. However, thorough phylogenetic comparisons (FIG. 2b) have shown that all mycolactone producers are closely related to M. ulcerans and have evolved from a common $M$. marinum progenitor to form a genetically cohesive group among a more diverse assemblage of $M$. marinum strains ${ }^{49}$. Like M. ulcerans, the fish and frog strains have a virulence plasmid and multiple copies of IS2404, but the pattern of DNA deletion and pseudogene accumulation seems to be different: only those strains that cause BU had reduced genomes. Comparisons of plasmid and chromosomal gene sequences show that plasmid acquisition, and the subsequent ability to produce mycolactone, was probably the key driver of speciation. As these mycolactone producers then radiated around the world, ongoing evolution produced at least two genetically distinct ecotypes that can be broadly divided into those that typically cause disease in ectotherms, such as fish and frogs, and those that typically cause disease in endotherms, such as humans.

\section{Molecular target of mycolactone}

Despite efforts from many research groups, the molecular target of mycolactone and the mechanism used by the toxin to suppress immune cell functions remain mysterious. The structure of mycolactone resembles that of the immunosuppressive agents FK506 and rapamycin (BOX 1). However, mycolactone has different effects on dendritic cell (DC) and T-cell immunobiology, suggesting that the toxin might bind to a different receptor and interfere with distinct signalling pathways. A fluorescent derivative of mycolactone and a ${ }^{14} \mathrm{C}$-labelled form of the toxin were both found to accumulate in a time- and dose-dependent manner in the cytoplasm of treated cells ${ }^{79}$ (C.D., unpublished observations), which supports the idea that mycolactone diffuses passively into target cells to interact with a cytosolic receptor. So far, structure-function studies have been limited to natural mycolactones. Strategies for the generation of additional mycolactone variants are required to investigate further the molecular mechanism of mycolactone action. In addition, detoxified variants of mycolactone might compete with mycolactone for receptor binding, and could therefore constitute valuable functional inhibitors of toxin. These could eventually become novel anti-BU therapeutics. 
Inflammatory infiltrate White blood cells that leave the blood to infiltrate inflamed connective tissues.

\section{Suppression of immune cell functions}

Although the basis of mycolactone cytotoxicity remains obscure, our understanding of the mechanism of mycolactone-induced immunosuppression has progressed. However, drawing conclusions on the type of immune response that is mounted in humans against $M$. ulcerans infection is rendered difficult by apparently contradictory results. In vitro studies show unambiguously that non-toxic doses of mycolactone are immunosuppressive on professional antigen-presenting cells. The most striking finding is the complete inhibition of tumour necrosis factor (TNF) production by monocytes and macrophages following infection with M. ulcerans or incubation with exogenous mycolactone $e^{9,25,80}$. Notably, bacterial production of mycolactone was also found to suppress the capacity of DCs to prime cellular responses and produce chemotactic signals that are crucial for inflammatory responses $^{81}$ (FIC. 4). This selective effect of mycolactone on the ability of immature DCs to secrete chemoattractants for monocytes and $\mathrm{T}$ helper $1\left(\mathrm{~T}_{H} 1\right)$ lymphocytes is in accordance with the histopathology of $\mathrm{BU}$, and supports the idea that local production of mycolactone in the skin prevents the trafficking of inflammatory cells to the ulcerative lesion.

However, these results seem to contradict quantitative studies of intralesional mRNA which indicate that the innate immune system is activated at the site of $\mathrm{BU}$ lesions, as shown by high mRNA levels for the cytokines interferon- $\gamma($ IFN- $\gamma)$, interleukin-1 $\beta$ (IL-1 $\beta)$, IL-6, IL-10, IL-12, IL-15 and TNF, and the chemokine IL-8 (REFS 7,82,83). There are several possible, non-exclusive explanations for this discrepancy between in vitro and in vivo observations. Results from mouse studies ${ }^{84}$ indicate that the lack of inflammatory infiltrates in BU is caused by the continuous destruction of inflammatory cells, rather than local immunosuppression. Alternatively, mycolactone or some other M. ulcerans component, may suppress the expression of inflammatory cytokines

\section{Box 1 | Parallels between mycolactone and other immunosuppressors}

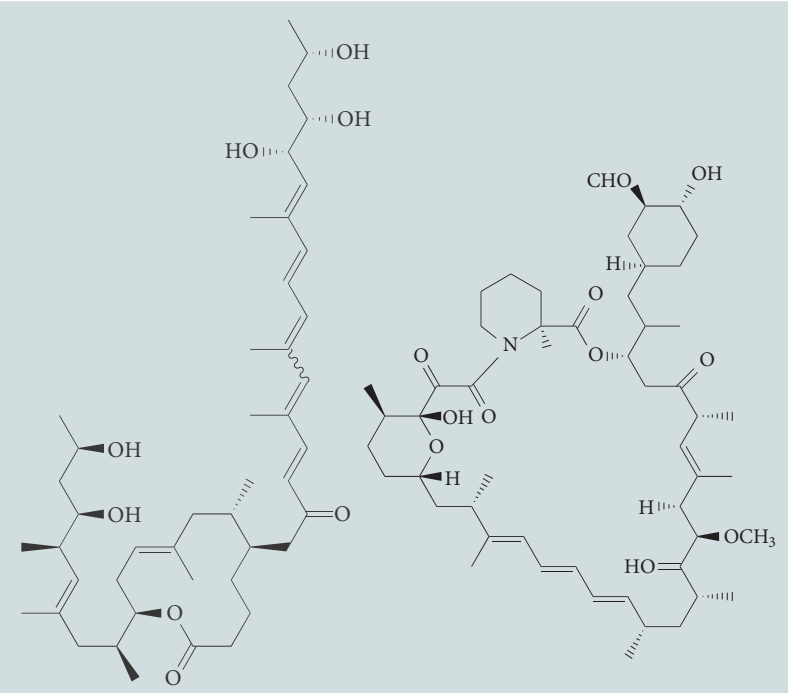

Mycolactone A and B

Origin Mycobacterium ulcerans

Molecular target ?

Mode of action ?

Effect on DCs

$\downarrow$ Co-stimulatory molecules

$\downarrow$ IL-6 and IL-12 No effect on TNF or IL-8

$\downarrow \beta$-chemokines

Effects on LTs

$\downarrow$ IL-2 and IFN- $\gamma$ Variable cytostatic effect
Rapamycin

S. hygroscopius

FKBPIA

mTOR inhibition

$\downarrow$ Co-stimulatory molecules

$\downarrow$ IL-12

$\downarrow$ Protein synthesis

Cell-cycle arrest

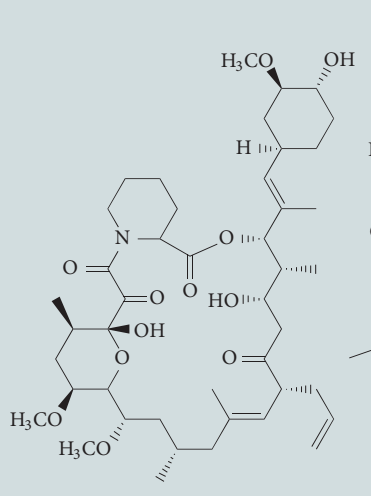

O=

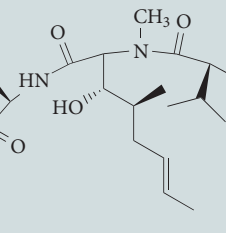

(1)

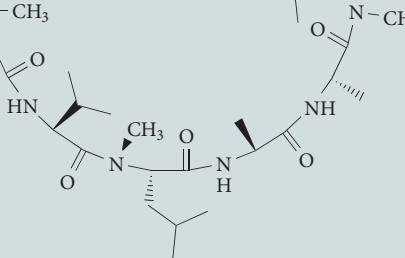

FK506

Cyclosporin A

S. tsukubaensis

Tolypocladium inflatum

FKBPIA

Calcineurin inhibition

Cyclophilin (immunophilin)

$\downarrow$ Co-stimulatory molecules

$\downarrow$ IL-6, IL-12, IL-8 and TNF

Calcineurin inhibition

$\downarrow \beta$-chemokines

$\downarrow$ Co-stimulatory molecules

$\downarrow$ IL-6, IL-12 and TNF

$\downarrow$ IL-2 and IFN- $\gamma$

$\downarrow$ IL-2, IFN- $\gamma$ and IL-4
Intriguingly, the structure of mycolactone is related to that of a family of natural products produced by actinomycetes ${ }^{15}$. In particular, it shares similar features with rapamycin, a macrocyclic triene produced by Streptomyces hygroscopicus and FK506, a macrolide lactone from Streptomyces tsukubaensis (see the figure). The molecular structure of the soil fungi metabolite cyclosporin $\mathrm{A}$ is more distant, but has a similar size and a similar proportion of hydrophobic groups ${ }^{81}$. FK506, rapamycin and cyclosporin $\mathrm{A}$ are all potent immunosuppressive drugs that alter the functional biology of lymphocytes and dendritic cells ${ }^{99}$. FK506 and rapamycin bind the same intracellular receptor, FKBP1A, although the resulting complex targets a different molecule. By contrast, FK506 and cyclosporin A bind different targets, but they both act by inhibiting calcineurin activity and produce similar biological effects. Similarly to mycolactone, FK506 modulates the production of $\beta$-chemokines. However, FK506 blocks dendritic cell production of inflammatory cytokines that are not altered by mycolactone, suggesting that mycolactone binds a different receptor and interferes with another signalling pathway that remains to be identified ${ }^{100}$. IFN, interferon; IL, interleukin; mTOR, mammalian target of rapamycin; TNF, tumour necrosis factor. 


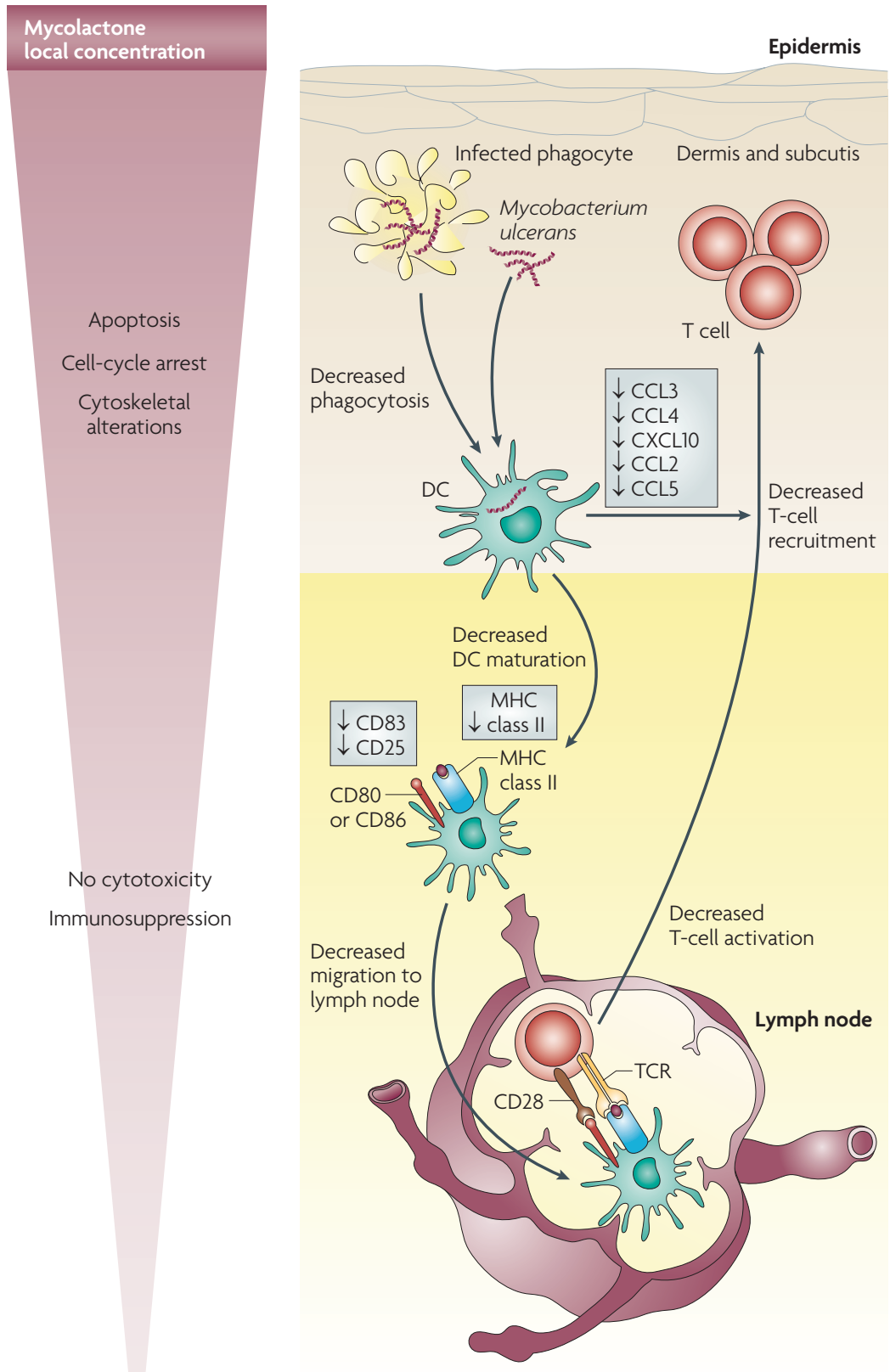

Figure 4 | Model of the cytotoxic and immunosuppressive actions of mycolactone in vivo. The triangle illustrates the gradient of mycolactone concentration from infectious foci in the skin to internal tissues, and the associated biological effects. The multiple immunosuppressive properties of mycolactone on dendritic cells (DCs) are shown, and their consequences on the initiation of primary immune responses and recruitment of inflammatory cells to the site of infection indicated ${ }^{81}$. CCL, C-C motif chemokine; CXCL10, C-X-C motif chemokine 10; MHC, major histocompatibility complex, TCR, T-cell receptor.
Several independent studies have reported modulation of the systemic IFN- $\gamma$ responses in patients with BU using restimulation assays of peripheral blood mononuclear cells ex $v i v o^{83,85-90}$. One of these studies reported an inverse ratio of IFN- $\gamma$ versus IL-10 in patients with ulcerative disease compared with subjects at the nodular stage $^{83}$. Although the IFN- $\gamma$ responses of patients with BU were not always significantly different to those of healthy controls, they were lower at the early stages compared with the ulcerative or healing stages ${ }^{88,89,91}$. Cases of disseminated disease have been reported in patients with AIDS, and there is an increased prevalence of HIV-1 or HIV-2 in patients with BU. Importantly, the systemic suppression of IFN- $\gamma$ responses in patients with $\mathrm{BU}$ is not specific for mycobacterial antigens, and resolves after surgical excision of the lesion ${ }^{90}$. In mice, cellular response defects occur following infection with wildtype $M$. ulcerans, but not with a mycolactone-deficient mutant ${ }^{95}$. Although there is no definitive evidence that mycolactone circulates in humans, it seems likely that the toxin is responsible for this systemic immunosuppression. In support of this idea, we found that subcutaneously delivered mycolactone diffuses into the peripheral blood of mice, and accumulates in internal organs with a tropism for lymphoid organs ${ }^{95}$. Furthermore, mycolactone is a potent suppressor of IL-2 and IFN- $\gamma$ production by human $\mathrm{T}$ cells in vitro ${ }^{96}$ (C.D., unpublished observations).

The generation of appropriate IFN- $\gamma$ responses is crucial for protective immunity against most mycobacterial infections. In BU, spontaneous healing often coincides with conversion to a positive delayed hypersensitivity against $M$. ulcerans antigens, suggesting that $\mathrm{T}_{\mathrm{H}} 1$ cellular immune responses are beneficial and contribute to the eradication of bacilli ${ }^{97}$. Interestingly, antibiotic treatment of BU induces vigorous inflammation in different compartments of the $\mathrm{skin}^{98}$, indicating that patients can mount $\mathrm{T}_{\mathrm{H}} 1$ responses. During the disease, the generation of cellular immunity can thus be suppressed by mycolactone in two ways (FIG. 4): first, at the site of infection, where the mycolactone concentration is cytotoxic, by killing resident DCs and inflammatory infiltrates; and second, at the systemic level, by reducing the ability of DCs and T cells to respond to stimulation without any major impact on their viability. In this model, withholding the immunosuppression imposed by mycolactone using inhibitors of its biosynthesis, or ablating its biological activity in vivo, would be sufficient to trigger the development of cellular immunity and allow the host immune system to control the infection.

\section{Concluding remarks}

Although there has been impressive recent progress in our exploration of the role of aquatic insects in the transmission of BU, our understanding of the precise mechanisms that occur remains incomplete. The coming years will reveal whether insects truly act as disease vectors or if they have simply been incriminated by their association with $M$. ulcerans. The highly sensitive molecular tools now available for tracking the BU bacillus will find increasing application and, like other genome-derived 
approaches, help to pinpoint the environmental source of infection. The history of BU provides a cautionary tale for other emerging diseases, as human intervention in the environment has clearly favoured emergence of the disease through the creation of new niches and habitats both for M. ulcerans and the aquatic insects within which it resides. Acquisition of the virulence plasmid by an ancestral M. marinum species though horizontal gene transfer was the main driver for disease emergence in humans and probably also for the infection of lower life forms. Unravelling the immunosuppressive pathways induced by mycolactone in mammalian cells will certainly be a profitable area of research, and improved understanding of the BU structure-activity relationship may enable the dissociation of cytotoxicity from immunosuppression. In turn, it would be satisfying if a version of a once disfiguring toxin could be engineered to afford therapeutic benefits similar to those of rapamycin to humans.
Alsop, D. The Bairnsdale ulcer. Aust. NZ J. Surg. 41, 317-319 (1972)

2. MacCallum, P., Tolhurst, J., Buckle, G. \& Sissons, H. A new mycobacterial infection in man. J. Pathol. Bacteriol. 60, 93-122 (1948)

This paper describes the discovery of M. ulcerans as the aetiological agent of Buruli ulcer disease.

3. Johnson, P. D. et al. Buruli ulcer (M. ulcerans infection): new insights, new hope for disease control. PLoS Med. 2, e108 (2005).

4. Marsollier, L. et al. Impact of Mycobacterium ulcerans biofilm on transmissibility to ecological niches and Buruli ulcer pathogenesis. PLoS Pathog. 3, e62 (2007).

Showed that $M$. ulcerans produces a mycolactone-rich extracellular matrix.

5. Guarner, J. et al. Histopathologic features of Mycobacterium ulcerans infection. Emerg. Infect. Dis. 9, 651-656 (2003).

6. Hayman, J. \& McQueen, A. The pathology of Mycobacterium ulcerans infection. Pathology 17, 594-600 (1985)

Excellent description of the pathology and histopathological features of $M$. ulcerans infection.

7. Peduzzi, E. et al. Local activation of the innate immune system in Buruli ulcer lesions. J. Invest. Dermatol. 127, 638-645 (2007).

8. Hayman, J. Clinical features of Mycobacterium ulcerans infection. Australas. J. Dermatol. 26, 67-73 (1985).

9. Coutanceau, E. et al. Modulation of the host immune response by a transient intracellular stage of Mycobacterium ulcerans: the contribution of endogenous mycolactone toxin. Cell. Microbiol. 7 1187-1196 (2005)

First description of $M$. ulcerans phagocytic uptake in vitro and intracellular transport in vivo. The study contradicted dogma that held the pathogen to be uniquely extracellular.

10. Torrado, E. et al. Evidence for an intramacrophage growth phase of Mycobacterium ulcerans. Infect. Immun. 75, 977-987 (2007).

11. Adusumilli, S. et al. Mycobacterium ulcerans toxic macrolide, mycolactone modulates the host immune response and cellular location of $M$. ulcerans in vitro and in vivo. Cell. Microbiol. 7, 1295-1304 (2005).

12. Connor, D. H. \& Lunn, H. F. Mycobacterium ulcerans infection (with comments on pathogenesis). Int J. Lepr. 33 (Suppl.), 698-709 (1965).

First suggestion that $M$. ulcerans can make a toxin

13. George, K. M. et al. Mycolactone: a polyketide toxin from Mycobacterium ulcerans required for virulence. Science 283, 854-857 (1999).

Landmark paper that presented the isolation of mycolactone and first demonstrated its crucial importance to $M$. ulcerans pathogenicity.

14. George, K. M., Pascopella, L., Welty, D. M. \& Small, P. L. A Mycobacterium ulcerans toxin, mycolactone, causes apoptosis in guinea pig ulcers and tissue culture cells. Infect. Immun. 68, 877-883 (2000).

15. Hong, H., Demangel, C., Pidot, S. J., Leadlay, P. F. \& Stinear, T. Mycolactones: immunosuppressive and cytotoxic polyketides produced by aquatic mycobacteria. Nature Prod. Rep. 25, 447-454 (2008).

Useful review of the different structures and activities of natural and bioengineered mycolactones.

16. Goto, M. et al. Nerve damage in Mycobacterium ulcerans-infected mice: probable cause of painlessness in Buruli ulcer. Am. J. Pathol. 168, 805-811 (2006).

17. En, J. et al. Mycolactone is responsible for the painlessness of Mycobacterium ulcerans infection (Buruli ulcer) in a murine study. Infect. Immun. 76, 2002-2007 (2008).
18. Diaz, D. et al. Use of the immunodominant 18-kiloDalton small heat shock protein as a serological marker for exposure to Mycobacterium ulcerans. Clin. Vaccine Immunol. 13, 1314-1321 (2006).

19. Etuaful, S. et al. Efficacy of the combination rifampinstreptomycin in preventing growth of Mycobacterium ulcerans in early lesions of Buruli ulcer in humans. Antimicrob. Agents Chemother. 49, 3182-3186 (2005).

20. Smith, P. G., Revill, W. D., Lukwago, E. \& Rykushin, Y. P. The protective effect of BCG against Mycobacterium ulcerans disease: a controlled trial in an endemic area of Uganda. Trans. R. Soc. Trop. Med. Hyg. 70, 449-457 (1977).

21. Huygen, K. Prospects for vaccine development against Buruli disease. Expert Rev. Vaccines 2, 561-569 (2003).

22. Coutanceau, E. et al. Immunogenicity of Mycobacterium ulcerans Hsp65 and protective efficacy of a Mycobacterium leprae Hsp65-based DNA vaccine against Buruli ulcer. Microbes Infect. 8, 2075-2081 (2006).

23. Tanghe, A., Content, J., Van Vooren, J. P., Portaels, F. \& Huygen, K. Protective efficacy of a DNA vaccine encoding antigen 85A from Mycobacterium bovis BCG against Buruli ulcer. Infect. Immun. 69, 5403-5411 (2001).

24. Tanghe, A., Dangy, J. P., Pluschke, G. \& Huygen, K. Improved protective efficacy of a species-specific DNA vaccine encoding mycolyl-transferase Ag85A from Mycobacterium ulcerans by homologous protein boosting. PLoS Negl. Trop. Dis. 2, e199 (2008).

25. Torrado, E. et al. Mycolactone-mediated inhibition of tumor necrosis factor production by macrophages infected with Mycobacterium ulcerans has implications for the control of infection. Infect. Immun. 75, 3979-3988 (2007).

26. Hayman, J. Postulated epidemiology of Mycobacterium ulcerans infection. Intern. J. Epidemiol. 20,

1093-1098 (1991).

27. Aiga, H. et al. Assessing water-related risk factors for Buruli ulcer: a case-control study in Ghana. Am. J. Trop. Med. Hyg. 71, 387-392 (2004).

28. Raghunathan, P. L. et al. Risk factors for Buruli ulcer disease (Mycobacterium ulcerans infection): results from a case-control study in Ghana. Clin. Infect. Dis. 40, 1445-1453 (2005)

29. World Health Organization. Buruli ulcer: history and background. (WHO, Geneva, 1975).

30. Marsollier, L. et al. Aquatic plants stimulate the growth of and biofilm formation by Mycobacterium ulcerans in axenic culture and harbor these bacteria in the environment. Appl. Environ. Microbiol. 70 , 1097-1103 (2004)

31. Marsollier, L. et al. Aquatic snails, passive hosts of Mycobacterium ulcerans. Appl. Environ. Microbiol. 70, 6296-6298 (2004).

32. Portaels, F., Elsen, P., Guimares-Peres, A., Fonteyne, P. A. \& Meyers, W. M. Insects in the transmission of Mycobacterium ulcerans infection. Lancet 353, 986 (1999).

First paper to suggest that insects play a part in the transmission of $M$. ulcerans from aquatic environments to humans.

33. Yip, M. J. et al. Evolution of Mycobacterium ulcerans and other mycolactone-producing mycobacteria from a common Mycobacterium marinum progenitor. J. Bacteriol. 189, 2021-2029 (2007).

34. Marsollier, L. et al. Aquatic insects as a vector for Mycobacterium ulcerans. Appl. Environ. Microbiol. 68, 4623-4628 (2002).

35. Marsollier, L. et al. Colonization of the salivary glands of Naucoris cimicoides by Mycobacterium ulcerans requires host plasmatocytes and a macrolide toxin, mycolactone. Cell. Microbiol. 7, 935-943 (2005).
36. Marsollier, L. et al. Early trafficking events of Mycobacterium ulcerans within Naucoris cimicoides Cell. Microbiol. 9, 347-355 (2007).

37. Marsollier, L. et al. Protection against Mycobacterium ulcerans lesion development by exposure to aquatic insect saliva. PLoS Med. 4, e64 (2007).

38. Portaels, F. et al. First cultivation and characterization of Mycobacterium ulcerans from the environment. PLoS Negl. Trop. Dis. 2, e1 78 (2008). First report of the isolation of $M$. ulcerans from the environment.

39. Johnson, P. D. et al. Mycobacterium ulcerans in mosquitoes captured during outbreak of Buruli ulcer, southeastern Australia. Emerg. Infect. Dis. 13 1653-1660 (2007).

First study to suggest that mosquitoes play a part in $M$. ulcerans transmission.

40. Debacker, M. et al. Mycobacterium ulcerans disease (Buruli ulcer) in rural hospital, Southern Benin 1997-2001. Emerg. Infect. Dis. 10, 1391-1398 (2004).

41. Debacker, M. et al. Mycobacterium ulcerans disease: role of age and gender in incidence and morbidity. Trop. Med. Int. Health 9, 1297-1304 (2004).

42. Quek, T. Y. et al. Risk factors for Mycobacterium ulcerans infection, southeastern Australia. Emerg. Infect. Dis. 13, 1661-1666 (2007).

43. Pouillot, R. et al. Risk factors for Buruli ulcer: a case control study in Cameroon. PLoS Negl. Trop. Dis. 1, e101 (2007).

44. Benbow, M. E. et al. Aquatic invertebrates as unlikely vectors of Buruli ulcer disease. Emerg. Infect. Dis. 14 1247-1254 (2008).

45. Tobin, D. M. \& Ramakrishnan, L. Comparative pathogenesis of Mycobacterium marinum and Mycobacterium tuberculosis. Cell. Microbiol. 10, 1027-1039 (2008).

46. Pozos, T. \& Ramakrishnan, L. New models for the study of Mycobacterium-host interactions. Curr. Opin. Immunol. 16, 499-505 (2004).

47. Ross, B. C. et al. Development of a PCR assay for rapid diagnosis of Mycobacterium ulcerans infection. J. Clin. Microbiol. 35, 1696-1700 (1997).

First description of the repeated element IS2404, the target for the gold-standard PCR method used for clinical diagnosis of $M$. ulcerans infection.

48. Stinear, T. et al. Identification and characterization of IS2404 and IS2606: two distinct repeated sequences for detection of Mycobacterium ulcerans by PCR. J. Clin Microbiol $37,1018-1023$ (1999).

49. Yip, M. J. et al. Evolution of Mycobacterium ulcerans and other mycolactone-producing mycobacteria from a common Mycobacterium marinum progenitor. J. Bacteriol. 189, 2021-2029 (2007).

50. Stinear, T. P., Jenkin, G. A., Johnson, P. D. R. $\delta$ Davies, J. K. Comparative genetic analysis of Mycobacterium ulcerans and Mycobacterium marinum reveals evidence of recent divergence. J. Bacteriol. 182, 6322-6330 (2000).

51. Portaels, F. et al. Variability in 3' end of $16 \mathrm{~S}$ rRNA sequence of Mycobacterium ulcerans is related to geographic origin of isolates. J. Clin. Microbiol. 34 962-965 (1996).

52. Rondini, S. et al. Ongoing genome reduction in Mycobacterium ulcerans. Emerg. Infect. Dis. 13 , 1008-1015 (2007).

53. Kaser, M. et al. Evolution of two distinct phylogenetic lineages of the emerging human pathogen Mycobacterium ulcerans. BMC Evol. Biol. 7, 177 (2007). 
54. Stinear, T. P. et al. Reductive evolution and niche adaptation inferred from the genome of Mycobacterium ulcerans, the causative agent of Buruli ulcer. Genome Res. 17, 192-200 (2007). Comprehensive description of the complete genome sequence of $M$. ulcerans

55. Parkhill, J. et al. Genome sequence of Yersinia pestis, the causative agent of plague. Nature $\mathbf{4 1 3}$ 523-527 (2001).

56. Parkhill, J. et al. Comparative analysis of the genome sequences of Bordetella pertussis, Bordetella parapertussis and Bordetella bronchiseptica. Nature Genet. 35, 32-40 (2003).

57. Abdallah, A. M. et al. Type VII secretion mycobacteria show the way. Nature Rev. Microbiol. 5, 883-891 (2007)

58. Brodin, P., Rosenkrands, I., Andersen, P., Cole, S. T. $\&$ Brosch, R. ESAT- 6 proteins: protective antigens and virulence factors? Trends Microbiol. 12 , 500-508 (2004)

59. Mishra, K. C. et al. Functional role of the PE domain and immunogenicity of the Mycobacterium tuberculosis triacylglycerol hydrolase LipY. Infect. Immun. 76, 127-140 (2008)

60. Fortune, S. M. et al. Mutually dependent secretion of proteins required for mycobacterial virulence. Proc. Natl Acad. Sci. USA 102, 10676-10681 (2005).

61. Huber, C. A., Ruf, M. T., Pluschke, G. \& Kaser, M Independent loss of immunogenic proteins in Mycobacterium ulcerans suggests immune evasion. Clin. Vaccine Immunol. 15, 598-606 (2008).

62. Reed, M. B. et al. A glycolipid of hypervirulent tuberculosis strains that inhibits the innate immune response. Nature 431, 84-87 (2004).

63. Daffe, M., Varnerot, A. \& Levy-Frebault, V. V. The phenolic mycoside of Mycobacterium ulcerans: structure and taxonomic implications. J. Gen. Microbiol. 138, 131-137 (1992).

64. Ramakrishnan, L., Tran, H. T., Federspiel, N. A. \& Falkow, S. A crtB homolog essential for photochromogenicity in Mycobacterium marinum isolation, characterization, and gene disruption via homologous recombination. J. Bacteriol. 179, 5862-5868 (1997)

65. Stinear T. P Pryor M. J., Porter J L \& Cole S. T. Functional analysis and annotation of the virulence plasmid pMUM001 from Mycobacterium ulcerans. Microbiology 151, 683-692 (2005).

66. Stinear, T. P. et al. Giant plasmid-encoded polyketide synthases produce the macrolide toxin of

Mycobacterium ulcerans. Proc. Natl Acad. Sci. USA 101, 1345-1349 (2004).

Landmark paper that described the genetic basis of mycolactone biosynthesis and the first detection of a virulence plasmid in mycobacteria.

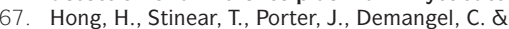 Leadlay, P. F. A novel mycolactone toxin obtained by biosynthetic engineering. Chembiochem 8 , 2043-2047 (2007).

68 Stinear, T. P. et al. Common evolutionary origin for the unstable virulence plasmid pMUM found in geographically diverse strains of Mycobacterium ulcerans. J. Bacteriol. 187, 1668-1676 (2005).

69. Mve-Obiang, A. et al. A newly discovered mycobacterial pathogen isolated from laboratory colonies of Xenopus species with lethal infections produces a novel form of mycolactone, the Mycobacterium ulcerans macrolide toxin. Infect. Immun. 73, 3307-3312 (2005).

70. Bali, S. \& Weissman, K. J. Ketoreduction in mycolactone biosynthesis: insight into substrate specificity and stereocontrol from studies of discret ketoreductase domains in vitro. Chembiochem 7 , 1935-1942 (2006)

71. Meier, J. L., Barrows-Yano, T., Foley, T. L., Wike, C. L. \& Burkart, M. D. The unusual macrocycle forming thioesterase of mycolactone. Mol. Biosyst. 4 663-671 (2008).

72. Tafelmeyer, P. et al. Comprehensive proteome analysis of Mycobacterium ulcerans and quantitative comparison of mycolactone biosynthesis. Proteomics 8, 3124-3138 (2008)

73. Ranger, B. S. et al. Globally distributed mycobacterial fish pathogens produce a nove plasmid-encoded toxic macrolide, mycolactone $\mathrm{F}$. Infect. Immun. 74, 6037-6045 (2006)

74. Mve-Obiang, A., Lee, R. E., Portaels, F. \& Small, P. L. Heterogeneity of mycolactones produced by clinical isolates of Mycobacterium ulcerans: implications for virulence. Infect. Immun. 71, 774-783 (2003).

75. Judd, T. C., Bischoff, A., Kishi, Y., Adusumilli, S. \& Small, P. L. Structure determination of mycolactone C via total synthesis. Org. Lett. 6, 4901-4904 (2004).

76. Hong, H., Stinear, T., Skelton, P., Spencer, J. B. \& Leadlay, P. F. Structure elucidation of a novel family of mycolactone toxins from the frog pathogen Mycobacterium sp. MU128FXT by mass spectrometry. Chem. Commun. (Camb.) 34 4306-4308 (2005)

77. Hong, H., Spencer J. B. Porter J L Leadlay, P. F \& Stinear, T. A novel mycolactone from a clinical isolate of Mycobacterium ulcerans provides evidence for additional toxin heterogeneity as a result of specific changes in the modular polyketide synthase. Chembiochem 6, 643-648 (2005). This paper describes how natural mycolactone variants are caused by polyketide synthase domain swapping.

78. Rhodes, M. W. et al. Mycobacterium pseudoshottsi sp. nov., a slowly growing chromogenic species isolated from Chesapeake Bay striped bass (Morone saxatilis). Int. J. Syst. Evol. Microbiol. 55, 1139-1147 (2005)

79. Snyder, D. S. \& Small, P. L. Uptake and cellular actions of mycolactone, a virulence determinant for Mycobacterium ulcerans. Microb. Pathog. 34 91-101 (2003)

80. Pahlevan, A. A. et al. The inhibitory action of Mycobacterium ulcerans soluble factor on monocyte/T cell cytokine production and NF-кB function. J. Immunol. 163, 3928-3935 (1999).

81. Coutanceau, E. et al. Selective suppression of dendritic cell functions by Mycobacterium ulcerans toxin mycolactone. J. Exp. Med. 204, 1395-1403 (2007).

Shows that mycolactone has the unique capacity to modulate the expression of $\beta$-chemokines by DCs.

82. Phillips, R. et al. Cytokine mRNA expression in Mycobacterium ulcerans-infected human skin and correlation with local inflammatory response. Infect. Immun. 74, 2917-2924 (2006).

83. Prevot, G. et al. Differential production of systemic and intralesional gamma interferon and interleukin-10 in nodular and ulcerative forms of Buruli disease. Infect. Immun. 72, 958-965 (2004)

84. Oliveira, M. S. et al. Infection with Mycobacterium ulcerans induces persistent inflammatory responses in mice. Infect. Immun. 73, 6299-6310 (2005).

85. Gooding, T. M. et al. Immune response to infection with Mycobacterium ulcerans. Infect. Immun. $\mathbf{6 9}$ 1704-1707 (2001)

86. Gooding, T. M., Johnson, P. D., Smith, M., Kemp, A. S $\&$ Robins-Browne, R. M. Cytokine profiles of patients infected with Mycobacterium ulcerans and unaffected household contacts. Infect. Immun. 70, 5562-5567 (2002)

87. Gooding, T. M., Kemp, A. S., Robins-Browne, R. M., Smith, M. \& Johnson, P. D. Acquired T-helper 1 lymphocyte anergy following infection with Mycobacterium ulcerans. Clin. Infect. Dis. 36 , 1076-1077 (2003)

88. Westenbrink, B. D. et al. Cytokine responses to stimulation of whole blood from patients with Buruli ulcer disease in Ghana Clin. Diagn. Lab. Immunol 12 125-129 (2005)

89. Phillips, R. et al. Cytokine response to antigen stimulation of whole blood from patients with Mycobacterium ulcerans disease compared to that from patients with tuberculosis. Clin. Vaccine Immunol. 13, 253-257 (2006).

90. Yeboah-Manu, D. et al. Systemic suppression of interferon-gamma responses in Buruli ulcer patients resolves after surgical excision of the lesions caused by the extracellular pathogen Mycobacterium ulcerans. J. Leukoc. Biol. 79, 1150-1156 (2006).

91. Kiszewski, A. E. et al. The local immune response in ulcerative lesions of Buruli disease. Clin. Exp. Immunol. $143,445-451$ (2006).
92. Bafende, A. E., Lukanu, N. P. \& Numbi, A. N. Buruli ulcer in an AIDS patient. S. Afr. Med. J. 92, 437 (2002).

93. Johnson, R. C. et al. Disseminated Mycobacterium ulcerans disease in an HIV-positive patient: a case study. AIDS 16, 1704-1705 (2002).

94 Johnson, R. C. et al. Association of HIV infection and Mycobacterium ulcerans disease in Benin. AIDS 22 901-903 (2008)

95. Hong, $\mathrm{H}$. et al. Mycolactone diffuses from Mycobacterium ulcerans-infected tissues and targets mononuclear cells in peripheral blood and lymphoid organs. PLoS Negl. Trop. Dis. 2, e325 (2008).

96. Hong, H., Stinear, T., Porter, J., Demangel, C. \& Leadlay, P. F. A novel mycolactone toxin obtained by biosynthetic engineering. Chembiochem 8 2043-2047 (2007)

97. Stanford, J. L., Revill, W. D., Gunthorpe, W. J. \& Grange, J. M. The production and preliminary investigation of Burulin, a new skin test reagent for Mycobacterium ulcerans infection. J. Hyg. (Lond.) 74 7-16 (1975)

98. Schutte, D. et al. Development of highly organized lymphoid structures in Buruli ulcer lesions after treatment with rifampicin and streptomycin. PLOS Negl. Trop. Dis. 1, e2 (2007).

99. Abe, M. \& Thomson, A. W. Influence of immunosuppressive drugs on dendritic cells. Transpl. Immunol. 11, 357-365 (2003)

100. Staruch, M. J., Camacho, R. \& Dumont, F. J. Distinctive calcineurin-dependent (FK506-sensitive) mechanisms regulate the production of the $\mathrm{CC}$ chemokines macrophage inflammatory protein (MIP)- $1 \alpha$, MIP- $1 \beta$, and RANTES vs IL- 2 and TNF- $\alpha$ by activated human T cells. Cell. Immunol. 190, 121-31 (1998).

101. Springer, B., Stockman, L., Teschner, K., Roberts, G. D. $\&$ Bottger, E. C. Two-laboratory collaborative study on identification of mycobacteria: molecular versus phenotypic methods. J. Clin. Microbiol. 34, 296-303 (1996)

102. Saitou, N. \& Nei, M. The neighbour-joining method: a new method for constructing phylogenetic trees. Mol. Biol. Evol. 4, 406-425 (1987)

103. Felsenstein, J. Confidence limits on phylogenies: an approach using the bootstrap. Evolution 39, 783-791 (1985)

104. Tamura, K., Dudley, J., Nei, M. \& Kumar, S. MEGA4: Molecular Evolutionary Genetics Analysis (MEGA) software version 4.0. Mol. Biol. Evol. 24, 1596-1599 (2007).

\section{Acknowledgements}

We thank the past and present members of our teams for their contributions. This work was supported in part by the National Health and Medical Research Council of Australia (to T.P.S.), the Agence Nationale pour la Recherche (to C.D.) and the Fondation Raoul Follereau (to S.T.C.).

\section{DATABASES}

Entrez Gene: http//www.ncbi.nlm nih gov/entrez/query. fcgi?db=gene

$\underline{\mathrm{crtl}}|\underline{\mathrm{mls} A 1}| \underline{\mathrm{mls}} \mathrm{s} 2|\underline{\mathrm{mlsB}}|$ MUL 1998

Entrez Genome Project: http://www.ncbi.nlm.nih.gov/ entrez/query.fcgi?db=genomepri

Bordetella pertussis | Mycobacterium bovis bacille CalmetteGuérin | Mycobacterium liflandii $\mid$ Mycobacterium tuberculosi |Mycobacterium ulcerans $\mid$ Yersinia pestis

UniProtKB: http://www.uniprot.or

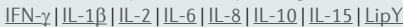

FURTHER INFORMATION

Caroline Demangel's homepage: http://www.pasteur.fr/ recherche/unites/Pmi

Stewart T. Cole's homepage: http://cole-lab.epfl.ch/ Timothy P. Stinear's homepage: http://www.med.monash. edu.au/microbiology/research/stinear.html BuruList World-Wide Web Server: http://genolist.pasteu fr/BuruList

WHO (secondary bacterial infection in M. ulcerans disease): http://www.who.int/buruli/information/diagnosis/en/ index6.html

ALL LINKS ARE ACTIVE IN THE ONLINE PDF 